\title{
LOCKING EFFECTS IN THE FINITE ELEMENT APPROXIMATION OF PLATE MODELS
}

\author{
MANIL SURI, IVO BABUŠKA, AND CHRISTOPH SCHWAB
}

\begin{abstract}
We analyze the robustness of various standard finite element schemes for a hierarchy of plate models and obtain asymptotic convergence estimates that are uniform in terms of the thickness $d$. We identify $h$ version schemes that show locking, i.e., for which the asymptotic convergence rate deteriorates as $d \rightarrow 0$, and also show that the $p$ version is free of locking. In order to isolate locking effects from boundary layer effects (which also arise as $d \rightarrow 0$ ), our analysis is carried out for the periodic case, which is free of boundary layers. We analyze in detail the lowest model of the hierarchy, the well-known Reissner-Mindlin model, and show that the locking and robustness of finite element schemes for higher models of the hierarchy are essentially identical to the Riessner-Mindlin case.
\end{abstract}

\section{INTRODUCTION}

The bending of elastic plates is often analyzed by replacing the actual 3-d plate problem by a 2-d model. It is possible to define a whole hierarchy of plate models, of which the well-known Reissner-Mindlin (RM) model is the lowest-order member, such that the modeling error can be controlled by using a sufficiently high-order model from the hierarchy. The numerical approximation of any plate model by the finite element method introduces an additional discretization error into the calculation. For certain finite element schemes, this discretization error can become very large when the thickness $d$ of the plate is close to zero. This occurs because of two phenomena, the existence of boundary layers and the presence of locking.

Boundary layers arise as components of the exact solution of the plate models and (to some extent) reflect the boundary layers present in the exact solution of the corresponding 3-d problem. In a series of papers (see [1] for references), Arnold and Falk have characterized in detail various aspects of the boundary layer for the exact RM solution, for different boundary conditions. (The boundary layers are in general different for different models of the hierarchy). One

Received by the editor May 18, 1992 and, in revised form, April 23, 1993 and December 17, 1993.

1991 Mathematics Subject Classification. Primary 65N30, 73V05.

Key words and phrases. Reissner-Mindlin, locking, finite element, $p$ version.

Research of the first and third authors was partially supported by the Air Force Office of Scientific Research, Air Force Systems Command, USAF, under Grants AFOSR 89-0252 and F49620-92-J0100 .

Research of the second author was partially supported by the U.S. Office of Naval Research under Grant N00014-J-1030. 
effect of the presence of these boundary layers is to weaken the a priori regularity of the solution. Since the singular behavior occurs only near the boundary, an effective strategy to overcome any consequent deterioration of numerical schemes is to locally refine the mesh (usually with a low-order scheme). See, for instance [2], where this has been done in the context of the $h-p$ version.

The second phenomenon mentioned above, numerical locking, also occurs when the thickness is close to zero, but for a different reason. It is well known that as the thickness $d \rightarrow 0$, the solutions of the plate models tend to the Kirchhoff plate solution. In the limiting case, the exact solution will therefore satisfy Kirchhoff's hypothesis. The problem of locking occurs because as $d \rightarrow 0$, the finite element solution is also forced to satisfy this hypothesis. Consequently, the number of conforming trial functions (which satisfy Kirchhoff's hypothesis) can get severely restricted, resulting in a degradation of the approximation properties of the trial space.

Since the problem of locking is quite different in terms of origin and numerical treatment from that of boundary layers, it is more instructive to analyze these problems separately. In this paper, we will be interested only in the problem of locking. To isolate this phenomenon and separate it from the effect of boundary layers, we will be considering the case of periodic boundary conditions, which we will choose so that the solution is smooth. (This also models the situation in the interior, in the case boundary layers are present.)

One method to avoid locking is to construct a finite element space which possesses optimal approximation properties even when restricted by Kirchhoff's constraint. This leads to a robust standard FEM, i.e., one whose performance is not sensitive to the thickness. An alternate strategy is to use a mixed method, which has the effect of enforcing the constraint in a weaker sense, thereby restricting the finite element space less. A number of mixed methods have been proposed and analyzed for the RM model, see e.g. [1, 10], Chapter 7 of [9] and the references therein.

Our approach in this paper will be to characterize the locking and robustness properties of various standard (as opposed to mixed) finite element schemes, for plate problems with periodic boundary conditions, using the general theory of locking developed by us in [4]. A key condition from that work, the so-called "condition $(\alpha)$ ", will be shown to be satisfied in this case, thereby reducing the question of locking to one of approximability alone. A similar technique was used by us in [5] to analyze Poisson ratio locking, which occurs in elasticity problems when the Poisson ratio is close to $1 / 2$. This technique is tailored to the first strategy mentioned above, i.e., the standard FEM, which involves the direct variational principle without reformulation. In contrast, mixed FEMs reformulate the variational principle and typically need compatible FE subspaces and an inf-sup approach for their analysis. The goal of this paper-and, of course, also a limitation-is to address only the standard variational method, which is directly usable for the entire hierarchy of plate models (such hierarchies also exist for laminated plates). As a consequence, our results apply to various elements from commercial codes based on the standard FEM. (We note that though we do not address mixed methods, some of the triangular finite elements analyzed by us in $\S 4$ in the context of the RM model can be formulated as equivalent mixed methods and, in fact, have been analyzed in this form as "Family III" of reference [10].) 
In $\S \S 2$ through 5 , we develop various results for the RM model, the lowestorder (and most commonly used) model in the hierarchy. For models higher up in the hierarchy, the asymptotic convergence in $d$ is faster, provided that the solution is smooth. The next highest model in the hierarchy after the RM (or $(1,1,0)$ ) model is the so-called $(1,1,2)$ plate model, which models the corner singularities and boundary layers of the 3-d problem more accurately than the RM model (see [2]). (Let us mention that for the clamped plate, the difference in data computed at the boundary from these two models can be as high as $30 \%$.)

In $\S 6$, we consider the question of locking for the $(1,1,2)$ model and, by extension, also for higher-order models in the hierarchy (once again for the periodic case). The techniques we use to analyze the RM case in $\S \S 2-5$ can be easily extended to such higher-order models as well (which is why we consider the RM case in such detail). We prove that the question of locking once again reduces to the Kirchhoff constraint being satisfied by the approximating subspaces. Hence, the results for the RM case carry over directly, with no additional locking effects being observed for higher-order models in our hierarchy.

Let us note that for the RM case, an alternative method of analysis based on the Helmholtz decomposition of the shear strain (see $[1,9,10])$ may be used. Most of the locking results we establish for the special RM case are, in fact, predictable from what is known for nearly incompressible elasticity ( $[5,13,14$, 16]) by this alternative method. See Remark 5.1 .

\section{The REISSNER-MindLin PLATE MODEL AND ITS REgUlARITY}

We consider as our domain the 2 -d midsection $\Omega=(-\pi, \pi)^{2}$ of a square isotropic plate with the plate occupying the region $R=\bar{\Omega} \times\left[-\frac{d}{2}, \frac{d}{2}\right]$. On $\Omega$ we consider the RM plate model for $u_{d}=\left(\vec{\phi}_{d}, \omega_{d}\right)$,

$$
\begin{gathered}
C_{d} u_{d}=-d^{2} \frac{D}{2}\left\{(1-\nu) \Delta \vec{\phi}_{d}+(1+\nu) \nabla \nabla \cdot \vec{\phi}_{d}\right\}-\kappa \mu\left(\nabla \omega_{d}-\vec{\phi}_{d}\right)=0 \\
-\kappa \mu d^{-2} \nabla \cdot\left(\nabla \omega_{d}-\vec{\phi}_{d}\right)=g
\end{gathered}
$$

which gives the bending of the plate in equilibrium. (Since the membrane effects do not exhibit shear locking, we consider in the present paper only the problem of pure bending.) We assume periodic boundary conditions,

$$
\begin{array}{rll}
\omega_{d}(x, \pi)=\omega_{d}(x,-\pi), & \omega_{d}(\pi, y)=\omega_{d}(-\pi, y), & |x|,|y| \leq \pi \\
(2.3 \mathrm{~b}) \vec{\phi}_{d}(x, \pi)=\vec{\phi}_{d}(x,-\pi), & \vec{\phi}_{d}(\pi, y)=\vec{\phi}_{d}(-\pi, y), & |x|,|y| \leq \pi
\end{array}
$$

Here, $\vec{\phi}_{d}$ gives the rotation of fibers normal to the midplane, $\omega_{d}$ measures the transverse displacement of the midplane, and $D=E / 12\left(1-\nu^{2}\right)$ is the flexural rigidity scaled by $d^{3}$. Also, $\mu=E / 2(1+\nu)$ is a Lamé coefficient, with $\kappa>0, E$ and $0 \leq \nu<0.5$ being the shear-correction factor, Young's modulus and Poisson ratio, respectively. (The second Lamé coefficient, $\lambda=$ $\nu E /(1+\nu)(1-2 \nu)$ will be used in $\S 6$.) Moreover, $g$ is the scaled loading function, i.e., the transverse load density per unit area divided by $d^{3}$. (We assume, essentially, that the loading function for a plate of thickness $d$ is $g d^{3}$, where $g$ is independent of $d$.) 
If we allow $d$ to tend to zero in (2.1), we formally obtain Kirchhoff's hypothesis,

$$
C_{0} u_{0}:=\vec{\phi}_{0}-\operatorname{grad} \omega_{0}=0 .
$$

The limiting solution $u_{0}=\left(\vec{\phi}_{0}, \omega_{0}\right)$ satisfies (2.4) together with

$$
D \Delta^{2} \omega_{0}=g
$$

and the periodic boundary conditions (2.3) (see, e.g., [3]). This, of course, is the classical biharmonic equation of plate bending, which (unlike the RM model) is independent of $d$ (and is sometimes used to model the actual 3-d plate).

For any domain $R \subset \mathbb{R}^{n}$ we will denote by $H^{s}(R)$ the usual Sobolev space with $s(=0,1,2, \ldots)$ square integrable derivatives. If $\tilde{f}$ denotes the $2 \pi$ periodic (in $x$ and $y$ ) extension of $f \in H^{s}(\Omega)$ to $\mathbb{R}^{n}$ (recall $\Omega=(-\pi, \pi)^{2}$ ), then $H_{\text {per }}^{s}(\Omega)$ will denote the set of those $f$ for which $\tilde{f} \in H_{\text {loc }}^{s}\left(\mathbb{R}^{n}\right)$. Using the method of real interpolation [7], we may define the above spaces for all real $s$. We will use $|\cdot|_{s},\|\cdot\|_{s}$ to denote the seminorm and norm, respectively, in both the periodic and nonperiodic case. We will also use $C_{\text {per }}^{(s)}(\Omega)$, which will denote the space of functions with $s$ periodic continuous derivatives.

Any $u(x)=u\left(x_{1}, x_{2}\right)$ in $H_{\mathrm{per}}^{s}(\Omega)$ can be expanded as a Fourier series,

$$
u(x)=\sum_{k \in \mathbb{Z}^{2}} u^{k} e^{i k \cdot x}, \quad k=\left(k_{1}, k_{2}\right), x=\left(x_{1}, x_{2}\right),
$$

where

$$
u^{k}=\frac{1}{4 \pi^{2}}\left\langle u, e^{-i k \cdot x}\right\rangle, \quad k \in \mathbb{Z}^{2},
$$

with $\langle\cdot, \cdot\rangle$ denoting the usual $L_{2}(\Omega)$ inner product. Then we have the following equivalences:

$$
|u|_{s}^{2} \sim \sum_{k \in \mathbb{Z}^{2}}|k|^{2 s}\left|u^{k}\right|^{2}, \quad\|u\|_{s}^{2} \sim \sum_{k \in \mathbb{Z}^{2}}\left(1+|k|^{2}\right)^{s}\left|u^{k}\right|^{2},
$$

which hold for all real $s \geq 0$. We may also use (2.7) to define the norms $\|u\|_{s}$, $s<0$, and the corresponding spaces $H_{\text {per }}^{s}(\Omega)$, which are the completions under these norms of $H_{\mathrm{per}}^{0}(\Omega)$.

We now cast (2.1)-(2.3) into the following weak form. For $d \in(0,1]$, find $u_{d}=\left(\vec{\phi}_{d}, \omega_{d}\right) \in V=\left[H_{\mathrm{per}}^{1}(\Omega)\right]^{3}$ such that for all $v=(\vec{\theta}, \xi) \in V$,

$$
a_{d}\left(u_{d}, v\right)=b\left(u_{d}, v\right)+\frac{\kappa \mu}{d^{2}}\left\langle C_{0} u_{d}, C_{0} v\right\rangle=F(v) .
$$

Here, $C_{0}$ is as defined in (2.4),

$$
b\left(u_{d}, v\right)=\frac{D}{2} \iint_{\Omega}\left\{(1-\nu) \nabla \vec{\phi}_{d} \cdot \nabla \vec{\theta}+(1+\nu)\left(\nabla \cdot \vec{\phi}_{d}\right)(\nabla \cdot \vec{\theta})\right\} d x_{1} d x_{2}{ }^{1}
$$

and

$$
F(v)=\langle g, \xi\rangle=\iint_{\Omega} g \xi d x_{1} d x_{2},
$$

with the last integral being understood as the pairing of $H_{\mathrm{per}}^{1}(\Omega)$ and $H_{\mathrm{per}}^{-1}(\Omega)$ if $g \in H_{\text {per }}^{-1}(\Omega)$. (For existence and uniqueness for this problem, see Theorem

'Our definition for $b(u, v)$ is equivalent to the usual one used for the RM plate model, since we have periodic boundary conditions. 
2.1 ahead.) We will denote the problem (2.8) by $P_{d}$ and assume, without loss of generality, that it is equivalent to (2.1)-(2.3).

We define the energy norm corresponding to $P_{d}$ by

$$
\|u\|_{E, d}^{2}=a_{d}(u, u), \quad u \in V .
$$

Also, for $u \in H_{\mathrm{per}}^{k}(\Omega) \times H_{\mathrm{per}}^{k}(\Omega) \times H_{\mathrm{per}}^{l}(\Omega)=: H_{\mathrm{per}}^{k}(\Omega)$, we let

$$
\|u\|_{k, l}^{2}=\|(\vec{\phi}, \omega)\|_{k, l}^{2}=\left\|\phi_{1}\right\|_{k}^{2}+\left\|\phi_{2}\right\|_{k}^{2}+\|\omega\|_{l}^{2},
$$

where the norm in $\omega$ is understood to be modulo constants. Then it may be shown that

$$
A_{1}\|u\|_{1,1} \leq\|u\|_{E, d} \leq A_{2} d^{-\beta}\|u\|_{1,1},
$$

where $A_{1}$ and $A_{2}$ are constants independent of $d$ and $\beta=1$. It is readily observed that for $d$ bounded away from 0 , the two norms are equivalent.

Let us now define, for $k \geq 1,0 \leq d \leq 1$, the spaces $H_{k, d} \subset H_{\text {per }}^{k+1, k}(\Omega)$ given by

$$
H_{k, d}=\left\{u \in H_{\mathrm{per}}^{k+1, k}(\Omega), C_{d} u=0\right\} .
$$

For any normed linear space $H$, we will denote the ball of radius $B>0$ by

$$
H^{B}=\left\{u \in H,\|u\|_{H} \leq B\right\} .
$$

We will use the notation

$$
H_{k}^{B}=H_{\mathrm{per}}^{k+1, k, B}(\Omega)=\left\{u \in H_{\mathrm{per}}^{k+1, k}(\Omega),\|u\|_{k+1, k} \leq B\right\}
$$

and

$$
H_{k, d}^{B}=H_{k}^{B} \cap H_{k, d} .
$$

We note that (2.10) may equivalently be characterized as

$$
H_{k, d}=\left\{u=(\vec{\phi}, \omega), \vec{\phi} \in H_{\mathrm{per}}^{k+1}(\Omega), C_{d} u=0\right\}
$$

since, by (2.1), it is easily seen that $\vec{\phi} \in H_{\mathrm{per}}^{k+1}(\Omega)$ implies $\omega \in H_{\mathrm{per}}^{k}(\Omega)$. Similarly, in the definition of $H_{k, d}^{B}$, we may replace $\|u\|_{k+1, k}$ by $\|\vec{\phi}\|_{k+1}$. We note also that for $u \in H_{k, d}^{B}$ we have, using (2.1),

$$
\left\|C_{0} u\right\|_{k-1} \leq K d^{2} B
$$

for $K$ a constant independent of $u, d$.

Let us look more closely at the limiting sets $H_{k, 0}$ and $H_{k, 0}^{B}$. Since for these $C_{0} u=0$, we see that $\vec{\phi} \in H_{\mathrm{per}}^{k+1}(\Omega)$ implies that $\nabla \omega=\vec{\phi} \in H_{\mathrm{per}}^{k+1}(\Omega)$, so that $\omega \in H_{\text {per }}^{k+2}(\Omega)$. Hence, we see that in this limiting case, the regularity of $\omega$ is increased by two derivatives, and we have the equivalent characterization

$$
\begin{aligned}
& H_{k, 0}=\left\{u \in H_{\mathrm{per}}^{k+1, k+2}(\Omega), C_{0} u=0\right\}, \\
& H_{k, 0}^{B}=\left\{u \in H_{k, 0},\|u\|_{k+1, k+2} \leq B\right\} .
\end{aligned}
$$

The choice of the above is motivated by the following theorem, which gives an a priori estimate in these weighted norms. 
Theorem 2.1. Let $g \in H_{\mathrm{per}}^{s-2}(\Omega), s \geq 1$, satisfy the compatibility condition

$$
\langle g, 1\rangle=0 \text {. }
$$

Then there exists a unique sequence of solutions $\left\{u_{d}\right\}=\left\{\vec{\phi}_{d}, \omega_{d}\right\} \quad\left(\omega_{d}\right.$ unique up to a constant $)$ to (2.1)-(2.3) for $d \in(0,1]$ and $(2.3)-(2.5)$ for $d=0$, such that $u_{d} \in H_{s, d}^{B}$, where $B=C\|g\|_{s-2}$, with $C$ a constant independent of $g$ and $d$.

Proof. Suppose $g, \phi_{d 1}, \phi_{d 2}$ and $\omega_{d}$ are represented in terms of their respective Fourier series, as in (2.6). Then (2.1)-(2.2) may be written as $(d>0)$

$$
\begin{array}{r}
\sum_{k \in \mathbf{Z}^{2}} e^{i k \cdot x}\left[\left(\begin{array}{ccc}
\frac{D}{2}\left\{2 k_{1}^{2}+(1-\nu) k_{2}^{2}\right\}+\kappa \mu d^{-2} & \frac{D}{2}(1+\nu) k_{1} k_{2} & -i \kappa \mu d^{-2} k_{1} \\
\frac{D}{2}(1+\nu) k_{1} k_{2} & \frac{D}{2}\left\{2 k_{2}^{2}+(1-\nu) k_{1}^{2}\right\}+\kappa \mu d^{2} & -i \kappa \mu d^{-2} k_{2} \\
i \kappa \mu d^{-2} k_{1} & i \kappa \mu d^{-2} k_{2} & \kappa \mu d^{-2}\left(k_{1}^{2}+k_{2}^{2}\right)
\end{array}\right)\right. \\
\\
\end{array}
$$

From this it may be easily verified that the solution $u_{d}$ of $(2.1)-(2.3)$ is

$$
u_{d}=\left(\begin{array}{c}
\phi_{d 1} \\
\phi_{d 2} \\
\omega_{d}
\end{array}\right)=\sum_{k \in \mathbf{Z}_{0}^{2}} e^{i k \cdot x}\left(\begin{array}{c}
\frac{i k_{1}}{D|k|^{4}} \\
\frac{i k_{2}}{D|k|^{4}} \\
\frac{1}{D|k|^{4}}+\frac{d^{2}}{\kappa \mu|k|^{2}}
\end{array}\right) g^{k},
$$

where $\mathbb{Z}_{0}=\mathbb{Z} \backslash\{0\}$. Here, we have used the fact that $g^{0}=0$, because of (2.18). Similarly, the solution of $(2.3)-(2.5)$ is given by

$$
\omega_{0}=\sum_{k \in \mathbf{Z}_{0}^{2}} e^{i k \cdot z} \frac{g^{k}}{D|k|^{4}},
$$

where $\vec{\phi}_{0}=\nabla \omega_{0}$. Note that in (2.19)-(2.20), $\omega_{d}$ is unique up to a constant.

From (2.19), it is easily seen that for $d>0$,

$$
\left\|u_{d}\right\|_{s+1, s} \leq C\|g\|_{s-2},
$$

proving that $u_{d} \in H_{s, d}^{B}$. The case $d=0$ follows similarly from (2.20).

We now prove the following theorem, which gives us the so-called "condition $(\alpha)$ " which is central to the locking theory developed in the next section. Essentially, this condition establishes the rate at which the solution of $(2.1)-(2.3)$ tends to the solution of $(2.3)-(2.5)$ in the $H_{\mathrm{per}}^{s+1, s}(\Omega)$ norm when $d$ is small.

Theorem 2.2. For any $u_{d}=\left(\vec{\phi}_{d}, \omega_{d}\right) \in H_{s, d}^{B}$, there exists $u_{0}=\left(\vec{\phi}_{0}, \omega_{0}\right) \in H_{s, 0}^{c B}$ such that

$$
\begin{gathered}
\vec{\phi}_{d}=\vec{\phi}_{0}, \\
\left\|\omega_{d}-\omega_{0}\right\|_{s} \leq K d^{2} B,
\end{gathered}
$$

where $c, K$ are constants independent of $u_{d}, d$ and $B$.

Proof. Given $u_{d}=\left(\vec{\phi}_{d}, \omega_{d}\right) \in H_{s, d}^{B}$, define $g$ (possibly depending on $d$ ) by (2.2). Then, by (2.2),

$$
\|g\|_{s-2} \leq K d^{-2}\left\|C_{0} u_{d}\right\|_{s-1} \leq K B
$$

where we have used (2.16). 
Now $\vec{\phi}_{d}, \omega_{d}$ may be expressed in terms of $g$ by (2.19). Also, let $\omega_{0}$ be given by $(2.20)$ and define $\vec{\phi}_{0}=\nabla \omega_{0}$. Then it is easily seen that $\vec{\phi}_{d}=\vec{\phi}_{0}$, and that $\left(\vec{\phi}_{0}, \omega_{0}\right) \in H_{s, 0}^{c B}$, where $c$ is independent of $u_{d}, d$ and $B$. Substituting $\vec{\phi}_{d}=\vec{\phi}_{0}=\nabla \omega_{0}$ in $(2.1)$, we have

$$
\nabla\left(\omega_{d}-\omega_{0}\right)=-\lambda^{-1} d^{2} \frac{D}{2}\left\{(1-\nu) \Delta \vec{\phi}_{d}+(1+\nu) \nabla \nabla \cdot \vec{\phi}_{d}\right\}
$$

from which it follows that

$$
\left\|\omega_{d}-\omega_{0}\right\|_{s} \leq K d^{2}\left\|\vec{\phi}_{d}\right\|_{s+1} \leq K d^{2} B
$$

The above theorem shows that for $d$ small, functions in $H_{s, d}^{B}$ are close to functions in $H_{s, 0}^{B}$. In this connection, we will also need the following result.

Theorem 2.3. Given $u_{0} \in H_{s, 0}^{B}, s \geq 1$, there exists a constant $C$ independent of $u_{0}, d$ such that for any $d \in(0,1]$, there is $a u_{d} \in H_{s, d}$ satisfying

$$
\left\|u_{d}\right\|_{E, d}+\left\|u_{d}\right\|_{s+1, s} \leq C\left\|u_{0}\right\|_{s+1, s+2}
$$

and

$$
\left\|u_{d}-u_{0}\right\|_{1,1} \rightarrow 0 \text { as } d \rightarrow 0
$$

Proof. First, using (2.16), one easily sees that for $s \geq 1$,

$$
\left\|u_{d}\right\|_{E, d} \leq C\left\|u_{d}\right\|_{s+1, s} .
$$

Next, let $u_{0}=\left(\operatorname{grad} \omega_{0}, \omega_{0}\right) \in H_{s, 0}^{B}$. Then, since $\omega_{0} \in H_{\mathrm{per}}^{s+2}(\Omega)$, we obtain the decomposition ( $\omega_{0}$ is defined modulo constants)

$$
\omega_{0}=\sum_{k \in \mathbb{Z}_{0}^{2}} e^{i k \cdot x} \omega^{k}, \quad \sum_{k \in \mathbb{Z}_{0}^{2}}\left(|k|^{2}\right)^{s+2}\left|\omega^{k}\right|^{2}<\infty .
$$

Hence, defining $g^{k}=D \omega^{k}|k|^{4}$, we see that

$$
g \in H_{\text {per }}^{s-2}(\Omega),\langle g, 1\rangle=0,\|g\|_{s-2} \leq\left\|\omega_{0}\right\|_{s+2} \leq C\left\|u_{0}\right\|_{s+1, s+2} .
$$

Using Theorem 2.1 together with (2.24)-(2.25) allows us to construct $u_{d}$ satisfying (2.21). Also, the argument of Theorem 2.2 shows that (2.22) holds.

\section{LOCKING AND ROBUSTNESS}

Suppose now that we are interested in approximating (2.8). We assume that we are given a sequence $\left\{V^{N}\right\}$ of finite-dimensional subspaces of $V=$ $\left[H_{\text {per }}^{1}(\Omega)\right]^{3}(N$ denoting the dimension, $N \in \mathscr{N})$. Then we can define the sequence of finite element solutions $u_{d}^{N} \in V^{N}$ by

$$
a_{d}\left(u_{d}^{N}, v\right)=a_{d}\left(u_{d}, v\right) \quad \forall v \in V^{N} .
$$

The sequence $\left\{V^{N}\right\}$ thus defines an extension procedure $\mathscr{F}$, i.e., a rule by which we can increase the dimension $N$ with the idea of increasing accuracy. Equation (3.1) immediately gives

$$
\left\|u_{d}-u_{d}^{N}\right\|_{E, d} \leq \inf _{v \in V^{N}}\left\|u_{d}-v\right\|_{E, d} .
$$


As shown in Theorem 2.1, depending upon the regularity of the data $g$, the exact solutions of our problem will belong to the sets $H_{k, d} \subset H_{\mathrm{per}}^{k+1, k}(\Omega)$, $k \geq 1$, introduced in $\S 2$. We will assume that the sequence $\left\{V^{N}\right\}$ is $F_{0^{-}}$ admissible, i.e., it leads to a certain fixed rate $F_{0}(N)$ of convergence when functions in $H_{\mathrm{per}}^{k+1, k}(\Omega)$ are approximated, in the following sense:

$$
A_{1} F_{0}(N) \leq \sup _{u \in H_{k}^{B}} \inf _{v \in V^{N}}\|u-v\|_{1,1} \leq A_{2} F_{0}(N) .
$$

Here, $F_{0}(N) \rightarrow 0$ as $N \rightarrow \infty$ and $A_{1}, A_{2}$ depend upon $B$ but are independent of $N$. Moreover, we assume that there exists $d_{0} \in(0,1)$ such that for $d_{0} \leq$ $d \leq 1$,

$$
A_{1}\left(d_{0}\right) F_{0}(N) \leq \sup _{u \in H_{k, d}^{B}} \inf _{v \in V^{N}}\|u-v\|_{1,1} \leq A_{2}\left(d_{0}\right) F_{0}(N)
$$

(Note that the lower bound in (3.3) follows from the one in (3.4), while the upper bound in (3.4) follows from the one in (3.3).)

Using (2.11), (3.2) and (3.4), we then obtain the following estimate, which holds uniformly for all $d_{0} \leq d \leq 1$ :

$$
\widetilde{A_{1}}\left(d_{0}\right) F_{0}(N) \leq \sup _{u_{d} \in H_{k, d}^{B}} E_{d}\left(u_{d}-u_{d}^{N}\right) \leq \widetilde{A_{2}}\left(d_{0}\right) F_{0}(N),
$$

where

$$
E_{d}(v)=\|v\|_{1,1} \text { or }\|v\|_{E, d} .
$$

Whether or not $\widetilde{A_{1}}, \widetilde{A_{2}}$ are bounded as $d_{0} \rightarrow 0$ will depend on the extension procedure being used.

A procedure $\mathscr{F}$ for which (3.5) holds uniformly for all $0<d \leq 1$ will be called free from locking for the sets $H_{k, d}$ with respect to the $E_{d}$ measure. A more precise definition, adapted from the general treatment of locking in [4], is given below.

Let $L(d, N)$, the locking ratio corresponding to $d \in(0,1], N \in \mathscr{N}$, with respect to the spaces $H_{k, d} \subset H_{\mathrm{per}}^{k+1, k}(\Omega)$ and error measures $\left\{E_{d}\right\}$ (as in (3.6)) for the problems (3.1), be defined by

$$
L(d, N)=\sup _{u_{d} \in H_{k, d}^{B}} E_{d}\left(u_{d}-u_{d}^{N}\right)\left(F_{0}(N)\right)^{-1} .
$$

Then we make the following definitions.

Definition 3.1. The extension procedure $\mathscr{F}$ is free from locking for the family of problems (3.1), $d \in(0,1]$, with respect to the solution sets $H_{k, d} \subset H_{\mathrm{per}}^{k+1, k}(\Omega)$ and error measures $E_{d}$ if and only if

$$
\limsup _{N \rightarrow \infty}\left[\sup _{d \in(0,1]} L(d, N)\right]=M<\infty .
$$

The extension procedure $\mathscr{F}$ shows locking of order $f(N)$ if and only if

$$
0<\limsup _{N \rightarrow \infty}\left[\sup _{d} L(d, N) \frac{1}{f(N)}\right]=K<\infty,
$$


where $F(N) \rightarrow \infty$ as $N \rightarrow \infty$. It shows locking of at least (respectively at most) order $f(N)$ if $K>0$ (respectively $K<\infty$ ).

Definition 3.2. The extension procedure $\mathscr{F}$ is robust for the family of problems (3.1), $d \in(0,1]$, with respect to the solution sets $H_{k, d} \subset H_{\mathrm{per}}^{k+1, k}(\Omega)$ and error measures $E_{d}$ if and only if

$$
\operatorname{Lim}_{N \rightarrow \infty} \sup _{d} \sup _{u_{d} \in H_{k, d}^{B}} E_{d}\left(u_{d}-u_{d}^{N}\right)=0 .
$$

It is robust with uniform order $g(N)$ if and only if

$$
\sup _{d} \sup _{u_{d} \in H_{k, d}^{B}} E_{d}\left(u_{d}-u_{d}^{N}\right) \leq g(N),
$$

where $g(N) \rightarrow 0$ as $N \rightarrow \infty$.

Remark 3.1. In $\S \S 4,5$ we will frequently use the form $g(N)=N^{-r}$ to characterize the robustness order $r$. If $r=0$, then convergence will not be guaranteed.

Definitions 3.1, 3.2 are related by the following theorem, from [4].

Theorem 3.1. The extension procedure $\mathscr{F}$ is free from locking if and only if it is robust with uniform order $F_{0}(N)$. Moreover, suppose $f(N)$ is such that

$$
f(N) F_{0}(N)=g(N) \rightarrow 0 \quad \text { as } N \rightarrow \infty .
$$

Then shows locking of order $f(N)$ if and only if it is robust with maximum uniform order $g(N)$.

It is easily seen that $\mathscr{F}$ is nonrobust if and only if it shows locking of order $\left(F_{0}(N)\right)^{-1}$.

Let us briefly explain the above ideas. We are assuming that our exact solution has a certain regularity (i.e., it is in $H_{\text {per }}^{k+1, k}(\Omega)$ ). Our extension procedure $\mathscr{F}$ has associated with it a rate of best approximation $F_{0}(N)$, which gives the best approximation that we could expect to achieve (equation (3.3)), for the most unfavorable exact solution in $H_{\mathrm{per}}^{k+1, k}(\Omega)$. Using the finite element method gives this rate for $d \geq d_{0}$ (equation (3.4)), but does not necessarily give this rate (uniformly) as $d$ approaches 0 . The locking ratio compares the accuracy actually achieved by the finite element method (for the least favorable exact solution in $H_{k, d}$ ) to the best accuracy possible (i.e., to $F_{0}(N)$ ). If the achieved accuracy is asymptotically the same as the rate $F_{0}(N)$ in (3.3), (3.4), uniformly for all $d \in(0,1]$, then we say that the extension procedure $\mathscr{F}=\left\{V^{N}\right\}$ is free of locking for all $u_{d} \in H_{k, d}^{B}, d \in(0,1]$. If the achieved accuracy is asymptotically not the same, then the robustness $g(N)$ gives the best rate of convergence that can be achieved independent of the parameter $d$. In this case, $f(N)=g(N)\left(F_{0}(N)\right)^{-1}$ characterizes the asymptotic strength of the locking.

In [4], we have formulated a useful condition, called condition $(\alpha)$, under which the question of locking reduces to one of approximability alone. For this condition to hold, we must first be given a sequence of solution spaces $H_{k, d}$ and a limit space $H_{k, 0}$ such that Theorem 2.3 holds. Then condition $(\alpha)$ may be stated as: Given $u_{d} \in H_{k, d}^{B}$, there exists a $u_{0} \in H_{k, 0}^{c B}$ (for some $c$ independent of $u_{d}, d, B ; u_{0}$ depending on $u_{d}$ ) such that

$$
\left\|u_{d}-u_{0}\right\|_{k+1, k} \leq K d^{\beta} B
$$


with $K$ a constant independent of $B, d$ and $u_{d}$. Here, $\beta$ is the exponent appearing in (2.11), $\beta=1$ in this case. This condition therefore characterizes the distance of solutions $u_{d}$ to functions $u_{0}$ satisfying (2.4), as $d \rightarrow 0$. We have shown in [4] that if the "remainder" $u_{d}-u_{0}$ is small in the sense of (3.7), then we need only consider the approximation of functions $u_{0}$ in the limit space $H_{k, 0}^{B}$ to answer questions about locking and robustness.

Our choice of periodic boundary conditions for the plate problem is motivated primarily by the fact that condition $(\alpha)$ is satisfied. To prove (3.7) for our problem, we simply choose $u_{0}$ (for given $u_{d}$ ) as in Theorem 2.2. Then we get (3.7); in fact, we get a power of $d^{2}$ (instead of just $d$, as needed). As a result, Theorem 2.4 from [4] will hold for our problem. This theorem states that locking and robustness rates are the same, no matter which error measure in (3.6) is used. It is stated below.

Theorem 3.2. Consider the family of problems $(3.1), d \in(0,1]$, with the solution sets $H_{k, d} \subset H_{\mathrm{per}}^{k+1, k}(\Omega), k \geq 1$. Then the extension procedure $\mathscr{F}$ is free from locking with respect to the $V=H_{\mathrm{per}}^{1,1}(\Omega)$ norm if and only if it is free with respect to the energy norm. It shows locking of order $f(N)$ in the $V$ norm if and only if it shows locking of order $f(N)$ in the energy norm.

Remark 3.2. We will now only refer to the locking of $\mathscr{F}$, without specifying the error measure in (3.6) we are using. Note, however, that instead of (3.6), we could have, in principle, defined some other error measure (for example, one that included the error in the shear strain). In that case, we would get different results, since the question of locking is extremely dependent upon the error functional under consideration (see $[4,5])$. The results in this paper only relate to the two error functionals in (3.6), i.e., we are assuming that the quantity we are interested in calculating is the energy, as opposed to some other quantity of interest.

The following theorem reduces the question of locking to one of approximability alone.

Theorem 3.3. Consider the problem (3.1) with solution sets $H_{k, d}, k \geq 1$. Let $V^{N}=Y^{N} \times Z^{N}$, where $Y^{N} \subset\left[H_{\mathrm{per}}^{1}(\Omega)\right]^{2}, Z^{N} \subset H_{\mathrm{per}}^{1}(\Omega)$, and define $W^{N} \subset Z^{N}$ by $W^{N}=\left\{\omega \in Z^{N}, \operatorname{grad} \omega \in Y^{N}\right\}$. Then the extension procedure $\mathscr{F}=\left\{V^{N}\right\}$ is robust with uniform order $\max \left\{F_{0}(N), g(N)\right\}$, where $g(N)$ is given by

$$
g(N)=\sup _{\omega \in H_{\mathrm{per}}^{k+2, B}} \inf _{z \in W^{N}}\|\omega-z\|_{2} .
$$

Also, with $F_{0}(N)$ as in (3.3), $\mathscr{F}$ is free from locking if and only if

$$
g(N) \leq C F_{0}(N) .
$$

It shows locking of order $f(N)$ if and only if

$$
C_{1} F_{0}(N) f(N) \leq g(N) \leq C_{2} F_{0}(N) f(N) .
$$

Proof. Define

$$
\tilde{g}(N)=\sup _{u \in H_{k, 0}^{B}} \inf _{v \in V^{n}}\|u-v\|_{1,1} .
$$

Since condition $(\alpha)$ is satisfied, by Theorem 2.2(B) of [4], $\mathscr{F}$ is robust with uniform order $\max \left\{F_{0}(N), \tilde{g}(N)\right\}$. The argument is as follows. For $u_{d} \in$ 
$H_{k, d}^{B}$, we may find (by condition $\left.(\alpha)\right)$ a $u_{0} \in H_{k, 0}^{B}$ such that (3.7) holds. Then we have

$$
\begin{aligned}
\left\|u_{d}-u_{d}^{N}\right\|_{1,1} & \leq\left\|u_{d}-u_{d}^{N}\right\|_{E, d} \leq \inf _{v \in V^{N}}\left\|u_{d}-v\right\|_{E, d} \\
& \leq \inf _{\substack{v_{1}+v_{2} \in V^{N} \\
C_{0} v_{1}=0}}\left\{\left\|u_{0}-v_{1}\right\|_{E, d}+\left\|\left(u_{d}-u_{0}\right)-v_{2}\right\|_{E, d}\right\} \\
& \leq \tilde{g}(N)+d^{-\beta} \inf _{v_{2} \in V^{N}}\left\|\left(u_{d}-u_{0}\right)-v_{2}\right\|_{1,1} \\
& \leq \tilde{g}(N)+d^{-\beta}\left[d^{\beta} F_{0}(N)\right],
\end{aligned}
$$

using (3.3) and (3.7) (the latter giving $\left.\left(u_{d}-u_{0}\right) \in H_{k}^{d B}\right)$. This proves the robustness order. Next, using the fact that Theorem 2.3 and condition $(\alpha)$ hold, we see by Theorem 2.2(B) of [4] that (3.9) and (3.10) will hold with $g(N)$ replaced by $\tilde{g}(N)$.

To show $g(N)$ and $\tilde{g}(N)$ are equivalent, consider a $v=(\vec{\psi}, z) \in V^{N}$ such that $C_{0} v=0$. Then

$$
C_{0} v=0 \Leftrightarrow z \in W^{N}, \quad \vec{\psi}=\operatorname{grad} z .
$$

Hence, for $u=(\vec{\phi}, \omega) \in H_{k, 0}^{B}$, we have

$$
\begin{aligned}
\tilde{g}(N) & =\sup _{u \in H_{k, 0}^{B}} \inf _{v \in V^{N}}\left\{\|\vec{\phi}-\vec{\psi}\|_{1}+\|\omega-z\|_{1}\right\} \\
& =\sup _{\omega \in H_{\text {per }}^{k+2}(\Omega)} \inf _{z \in W^{N}}\left\{\|\operatorname{grad} \omega-\operatorname{grad} z\|_{1}+\|\omega-z\|_{1}\right\},
\end{aligned}
$$

from which it is easily seen that $g(N)$ and $\tilde{g}(N)$ are equivalent. The theorem follows.

Let us now define the subspaces $V^{N}=Y^{N} \times Z^{N}$. We will consider both triangular and rectangular meshes. Our results will be for the uniform triangular and rectangular meshes $M_{1}^{h}, M_{2}^{h}$ shown for the case of our square domain $\Omega$ in Figure 3.1.

Let $S$ be a triangle or parallelogram. Then we define $\mathscr{P}_{p}^{1}(S)=$ polynomials on $S$ of total degree $\leq p$, $\mathscr{P}_{p}^{2}(S)=$ polynomials on $S$ of degree $\leq p$ in each variable, $\mathscr{P}_{p}^{3}(S)=\mathscr{P}_{p}^{1}(S) \oplus\left\{x_{1}^{p} x_{2}, x_{1} x_{2}^{p}\right\}$, the serendipity elements.
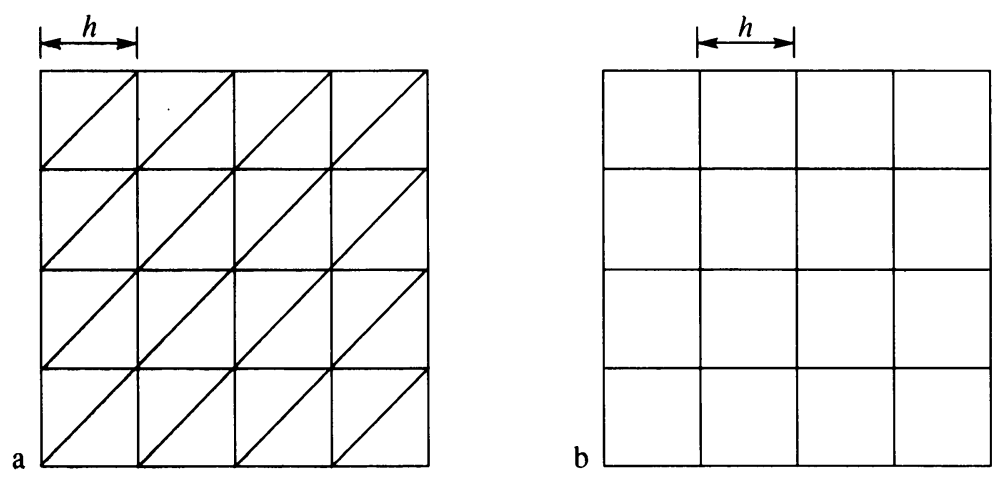

FIGURE 3.1. Uniform meshes $M_{1}^{h}, M_{2}^{h}$ 
Remark 3.3. The spaces $\mathscr{P}_{p}^{2}(S), \mathscr{P}_{p}^{3}(S)$ are the usual $\mathscr{Q}_{p}$ and $\mathscr{Q}_{p}^{\prime}$ spaces (and are defined only for $S$ a parallelogram).

For any mesh $M^{h}$, we now define for $i=1,2,3$

$$
\mathscr{P}_{p,-1}^{i, h}=\mathscr{P}_{p,-1}^{i, h}\left(M^{h}\right)=\left\{v \in L_{2}(\Omega),\left.v\right|_{\Omega_{j}^{h}} \in \mathscr{P}_{p}^{i}\left(\Omega_{j}^{h}\right)\right\},
$$

where $\Omega_{j}^{h}$ are the elements of $M^{h}$. For $k>0$, we define

$$
\mathscr{P}_{p, k}^{i, h}=\mathscr{P}_{p, k}^{i, h}\left(M^{h}\right)=\mathscr{P}_{p,-1}^{i, h} \cap C_{\mathrm{per}}^{(k)}(\Omega),
$$

where $\mathscr{P}_{p, k}^{2, h}$ and $\mathscr{P}_{p, k}^{3, h}$ are defined for rectangular meshes only. In the succeeding sections, we will consider the locking effects of the spaces $V^{N}=V_{p, q}^{i, h}$ defined by taking $Y^{N}=Y_{p}^{i, h}=\left[\mathscr{P}_{p, 0}^{i, h}\right]^{2}$ and $Z^{N}=Z_{q}^{i, h}=\mathscr{P}_{q, 0}^{i, h}$. Note that for the space $\mathscr{P}_{p, 0}^{i, h}$, one has $N=O\left(h^{-2} p^{2}\right)$.

\section{THE $h$ VERSION}

In this section, we consider the robustness of the finite element spaces $V^{N}=$ $V_{p, q}^{i, h}$ when $p, q$ are held fixed and $h$ is decreased to attain accuracy. We first estimate $F_{0}(N)$ in (3.3), (3.4).

Lemma 4.1. Consider an $h$ version sequence of spaces $\left\{V^{N}\right\}=\left\{V_{p}^{i, h}\right\}$ defined by $V_{p, q}^{i, h}=Y_{p}^{i, h} \times Z_{q}^{i, h}=\left[\mathscr{P}_{p, 0}^{i, h}\right]^{2} \times \mathscr{P}_{q, 0}^{i, h}$ on an appropriate triangular or parallelogram quasi-uniform mesh, where $p, q \geq 1$ are fixed and $h$ varies. Then for any $d_{0}>0,(3.4)$ is satisfied with

$$
F_{0}(N)=C N^{-\min (p, q, k-1) / 2},
$$

where $C$ is independent of $N$ but depends on $p, q, k$. Moreover, (3.3) is also satisfied with the above $F_{0}$.

Proof. We first note that for $l>1, r \geq 1$, with $\beta=\min (r, l-1) / 2$,

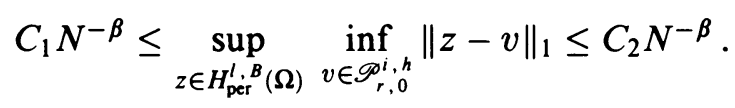

This has been shown in Theorem 4.1 of [5] (for example) for the nonperiodic case, from which the periodic case is easily deduced. Using (4.2), we easily see that the upper estimate in (3.3) (and hence (3.4)) holds with $F_{0}$ given by (4.1). To establish the lower estimate in (3.4) (and hence (3.3)), we note that given a periodic $\omega_{d}$ (or conversely a periodic $\vec{\phi}_{d}$ ), we can find a corresponding periodic function $\vec{\phi}_{d}$ (or a periodic $\tilde{\omega}_{d}$ ) satisfying (2.1) such that $u_{d}=\left(\vec{\phi}_{d}, \omega_{d}\right)$ (or $\tilde{u}_{d}=\left(\tilde{\vec{\phi}}_{d}, \tilde{\omega}_{d}\right)$ ) lies in $H_{k, d}^{B}$ (for appropriate $k$ ). Hence, (4.2) can be used to establish the required lower bound.

We may now analyze the locking and robustness properties of various types of elements by calculating $g(N)$ defined by (3.8) and comparing it with $F_{0}(N)$ given by (4.1). We first consider triangular elements.

Suppose that $V^{N}=V_{p, q}^{1, h}$ (as in Lemma 4.1) is defined on the mesh $M_{1}^{h}$. Then we see that

$$
\begin{aligned}
W^{N} & =\mathscr{P}_{q, 1}^{1, h}, & & q \leq p, \\
& =\mathscr{P}_{p+1,1}^{1, h}, & & q \geq p+1 .
\end{aligned}
$$


Let us define

$$
\begin{aligned}
r(q) & =\max (2, q) & & \text { for } 1 \leq q \leq 4, \\
& =q+1 & & \text { for } q \geq 5 .
\end{aligned}
$$

Then the following lemma follows from Theorem 5.1 and Lemma 5.2 of [5], where it has been established for the nonperiodic case.

Lemma 4.2. Let $M_{1}^{h}$ be the uniform triangular mesh of Figure 3.1. Then for $\omega \in H_{\mathrm{per}}^{k+2}(\Omega), k \geq 0, q \geq 1$,

$$
\inf _{z \in \mathscr{P}_{q, 1}^{1, h}}\|\omega-z\|_{2} \leq C h^{\min (k, r(q)-2)}\|\omega\|_{k+2} .
$$

Moreover, there exists a function $Q \in C_{\mathrm{per}}^{(\infty)}(\Omega)$ satisfying

$$
\inf _{z \in \mathscr{P}_{q, 1}^{1, h}}\|Q-z\|_{2} \geq C h^{r(q)-2} .
$$

We can now prove the following theorem.

Theorem 4.1. Let the extension procedure $\mathscr{F}$ consist of the $h$ version on the uniform triangular mesh $M_{1}^{h}$, with spaces $\left\{V^{N}\right\}=\left\{V_{p, q}^{1, h}\right\}=\left\{\left[\mathscr{P}_{p, 0}^{1, h}\right]^{2} \times \mathscr{P}_{q, 0}^{1, h}\right\}$. Let the solution sets be $H_{k, d}$. Then for the $p$ and $q$ shown below, $\mathscr{F}$ is robust with uniform order $N^{-r}$ when $k \geq 2 r+1$ and shows locking of order $N^{l}$ when $k \geq p+1$.

\begin{tabular}{|r|c|c|c|}
\hline $\begin{array}{c}\text { Degree } \\
p\end{array}$ & $\begin{array}{c}\text { Degree } \\
q\end{array}$ & $\begin{array}{c}\text { Robustness } \\
\text { order }=O\left(N^{-r}\right)\end{array}$ & $\begin{array}{c}\text { Locking order } \\
=O\left(N^{l}\right)\end{array}$ \\
\hline \hline 1 & $q \geq 1$ & 0 & $l$ \\
\hline $2 \leq p \leq 4$ & $q=p$ & $(p-2) / 2$ & $1 / 2$ \\
$p \geq 5$ & & $(p-1) / 2$ & 1 \\
\hline $2 \leq p \leq 3$ & $q \geq p+1$ & $(p-1) / 2$ & $1 / 2$ \\
$p \geq 4$ & $q \geq p+1$ & $p / 2$ & 0 \\
\hline
\end{tabular}

(For $p>q$, the same results as for the case $(p, q)=(q, q)$ hold.)

Remark 4.1. The above theorem shows that with the customary choice $p=q$, convergence is not guaranteed for $p=1$ or 2 (i.e., the robustness order $r$ is 0 ) and is only guaranteed (with reduced order) if $p \geq 3$. In fact, locking cannot be avoided whenever we take $p=q$. It is $O(N)$ for $p=2,3,4$ and $O\left(N^{1 / 2}\right)$ for $p \geq 5$ (for $p=1$, it is technically $O\left(N^{1 / 2}\right)$ as well, since the maximum possible rate in this case is $\left.O\left(N^{-1 / 2}\right)\right)$. To avoid locking, $p$ has to be taken to be 4 or larger, with $q$ being chosen to be $p+1$. (Note that taking $q>p+1$ will not increase the robustness rate.)

Proof of Theorem 4.1. We illustrate the proof for $p=2$, for the two cases $q=2, q \geq 3$. By Lemma 4.1, we see that for both cases, for $k \geq 1$,

$$
F_{0}(N)=C N^{-\min (2, k-1) / 2}
$$


and for $k \geq 3$ (i.e., $k \geq p+1$ ), we have the best rate that we can expect, i.e., $O\left(N^{-1}\right)$. We now calculate the robustness rate actually achieved, given by $\max \left(F_{0}(N), g(N)\right)$, where $g(N)$ is defined by (3.8).

For $q=2$, we see that, using (4.3), we have

$$
g(N)=\sup _{\omega \in H_{\mathrm{per}}^{k+2, B}(\Omega)} \inf _{z \in \mathscr{P}_{2,1}^{1, h}}\|\omega-z\|_{2} .
$$

By Lemma 4.2, for any $k \geq 0$, we see that

$$
C_{1} \leq g(N) \leq C
$$

and hence, for $k \geq 1$, the robustness rate is $\max \left(F_{0}(N), g(N)\right) \sim C$. Hence, this method is not robust. By (4.4), for $k \geq 3, F_{0}(N)=C N^{-1}$, so that by Theorem 3.3, the locking is $O\left(N^{1}\right)$.

For $q \geq 3$, we get (4.5) again, except $z$ is now in $\mathscr{P}_{3,1}^{1, h}$. By Lemma 4.2, for $k \geq 1$,

$$
C_{1} h \leq g(N) \leq C_{2} h,
$$

i.e., $g(N) \sim C N^{-1 / 2}$. Using (4.4), we see that for $k \geq 2, F_{0}(N) \leq C N^{-1 / 2}$, so that the robustness rate is $\max \left(F_{0}(N), g(N)\right)=C N^{-1 / 2}$ for $k \geq 2$. Also, for $k \geq 3, F_{0}(N)=C N^{-1}$, and by Theorem 3.3, for $k \geq 3$, the locking is $O\left(N^{1 / 2}\right)$.

Remark 4.2. The robustness for the case $p \geq 4, q=p+1$, was established in [10] by a different method. It was, in fact, shown that this case is locking-free even when the quasi-uniform version of $M_{1}^{h}$ is used. As discussed ahead in Remark 5.1, the results of Theorem 4.1 can be deduced from the results in [5, $13,14]$ for nearly incompressible elasticity.

Let us now consider the uniform rectangular mesh $M_{2}^{h}$. Suppose first that the extension $\mathscr{F}$ is based on $\mathscr{P}_{p}^{2}(S)$ elements, with $V^{N}=V_{p, q}^{2, h}=\left[\mathscr{P}_{p, 0}^{2, h}\right]^{2} \times \mathscr{P}_{q, 0}^{2, h}$. Then it may be observed that

$$
\begin{aligned}
W^{N} & =\mathscr{P}_{q, 1}^{2, h}, & & q \leq p, \\
& =\mathscr{P}_{p+1,1}^{1, h} \cup \mathscr{P}_{p, 1}^{2, h}, & & q \geq p+1 .
\end{aligned}
$$

The following is an analog of Lemma 4.2 for this case. The nonperiodic version of this result is established in $[8,5]$.

Lemma 4.3. Let $M_{2}^{h}$ be the uniform rectangular mesh of Figure 3.1. Then for $\omega \in H_{\mathrm{per}}^{k+2}(\Omega), k \geq 0, q \geq 1$,

$$
\inf _{z \in \mathscr{P}_{q, 1}^{2, h}}\|\omega-z\|_{2} \leq C h^{\min (k, q-1)}\|\omega\|_{k+2} .
$$

Moreover, there exists a function $Q \in C_{\mathrm{per}}^{(\infty)}(\Omega)$ satisfying

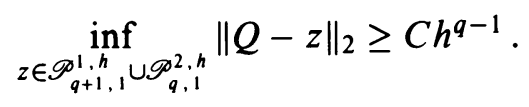

Obviously, the two bounds (4.7) and (4.8) hold for both the spaces $\mathscr{P}_{q+1,1}^{1, h} \cup$ $\mathscr{P}_{q, 1}^{2, h}$ and $\mathscr{P}_{q, 1}^{2, h}$. Now using Theorem 3.3, Lemma 4.1, (4.6) and Lemma 4.3, we obtain the following theorem, whose proof is similar to that of Theorem 4.1. 
Theorem 4.2. Let the extension procedure $\mathscr{F}$ consist of the $h$ version on the uniform rectangular mesh $M_{2}^{h}$, with spaces $\left\{V^{N}\right\}=\left\{V_{p, q}^{2, h}\right\}=\left\{\left[\mathscr{P}_{p, 0}^{2, h}\right]^{2} \times \mathscr{P}_{q, 0}^{2, h}\right\}$. Let the solution sets be $H_{k, d}$. Then for the $p$ and $q$ shown below, $\mathscr{F}$ is robust with uniform order $N^{-r}$ when $k \geq 2 r+1$ and shows locking of order $N^{l}$ when $k \geq p+1$.

\begin{tabular}{|c|c|c|c|}
\hline $\begin{array}{c}\text { Degree } \\
p\end{array}$ & $\begin{array}{c}\text { Degree } \\
q\end{array}$ & $\begin{array}{c}\text { Robustness } \\
\text { order }=O\left(N^{-r}\right)\end{array}$ & $\begin{array}{c}\text { Locking order } \\
=O\left(N^{l}\right)\end{array}$ \\
\hline \hline 1 & $q \geq 1$ & 0 & $l$ \\
\hline$p \geq 2$ & $q \geq p$ & $(p-1) / 2$ & $1 / 2$ \\
\hline
\end{tabular}

We now consider an extension procedure on $M^{h}$ based on $\mathscr{P}^{3}(S)$-type elements. Accordingly, suppose $V^{N}=V_{p, q}^{3, h}$. Then it can be shown that

$$
\begin{aligned}
W^{N} & =\mathscr{P}_{q, 1}^{3, h}, & & q \leq p, \\
& =\mathscr{P}_{p+1,1}^{1, h}, & & p \neq 2, q \geq p+1, \text { or }(p, q)=(2,3), \\
& =\mathscr{P}_{3,1}^{1, h} \cup \mathscr{P}_{2,1}^{2, h}, & & p=2, q \geq 4 .
\end{aligned}
$$

In this case, we have the following lemma.

Lemma 4.4. Let $M_{2}^{h}$ be the uniform rectangular mesh of Figure 3.1. Then for $\omega \in H_{\text {per }}^{k+2}(\Omega), k \geq 0, q \geq 1$

$$
\inf _{z \in \mathscr{P}_{q, h}^{1, h}}\|\omega-z\|_{2} \leq C h^{\min (k, m(q))}\|\omega\|_{k+2},
$$

where $m(q)=\max (0, q-3)$. Moreover, there exists a function $Q \in C_{\mathrm{per}}^{(\infty)}(\Omega)$ satisfying

$$
\inf _{z \in \mathscr{P}_{q, 1}^{3, h}}\|Q-z\|_{2} \geq C h^{m(q)}
$$

Proof. The assertion (4.10) follows from Theorems 1, 2 of [8], as shown in [5]. Next, we note that in Lemma 5.2 of [5], (4.11) was established for $\mathscr{P}_{q, 1}^{1, h}$ instead of $\mathscr{P}_{q, 1}^{3, h}$, for the nonperiodic case. Essentially the same proof can be used to prove (4.11) here as well.

Note that the bounds in (4.10), (4.11) will hold for $\mathscr{P}_{q, 1}^{3, h}$ and $\mathscr{P}_{q, 1}^{1, h}$, respectively, as well. Using (4.9) and Lemma 4.4 , we may once again establish the following theorem, analogously to Theorem 4.1.

Theorem 4.3. Let the extension procedure $\mathscr{F}$ consist of the $h$ version on the uniform rectangular mesh $M_{2}^{h}$, with spaces $\left\{V^{N}\right\}=\left\{V_{p, q}^{3, h}\right\}=\left\{\left[\mathscr{P}_{p, 0}^{3, h}\right]^{2} \times \mathscr{P}_{q, 0}^{3, h}\right\}$. Let the solution sets be $H_{k, d}$. Then for the $p$ and $q$ shown below, $\mathscr{F}$ is robust with uniform order $N^{-r}$ when $k \geq 2 r+1$ and shows locking of order $N^{l}$ when $k \geq p+1$. 


\begin{tabular}{|c|c|c|c|}
\hline $\begin{array}{c}\text { Degree } \\
p\end{array}$ & $\begin{array}{c}\text { Degree } \\
q\end{array}$ & $\begin{array}{c}\text { Robustness } \\
\text { order }=O\left(N^{-r}\right)\end{array}$ & $\begin{array}{c}\text { Locking order } \\
=O\left(N^{l}\right) \\
l\end{array}$ \\
\hline \hline 1 & $q \geq 1$ & 0 & $1 / 2$ \\
\hline 2 & $q=2,3$ & 0 & 1 \\
& $q \geq 4$ & $1 / 2$ & $1 / 2$ \\
\hline$p \geq 3$ & $q=p$ & $(p-3) / 2$ & $3 / 2$ \\
& $q \geq p+1$ & $(p-2) / 2$ & 1 \\
\hline
\end{tabular}

Remark 4.3. Theorems 4.2 and 4.3 show that locking cannot be avoided when rectangular elements are used, no matter what choices of $p$ and $q$ are made. Both $\mathscr{P}_{p}^{2}$ and $\mathscr{P}_{q}^{3}$ elements are robust only when $p \geq 2$ (for $\mathscr{P}_{2}^{2}$, we only get robustness if, moreover, $q \geq 4$ ). For $p \geq 3, \mathscr{P}_{p}^{3}$ elements show twice to three times the locking rate as $\mathscr{P}_{p}^{2}$ elements, depending on the choice of $q$. Similar results have been established for rectangular elements in the context of nearly incompressible elasticity [5] (see Remark 5.1).

\section{THE $p$ AND $h-p$ VERSIONS}

We now consider a $p$ version extension procedure $\mathscr{F}$, with $\left\{V^{N}\right\}=\left\{V_{p, q}^{i, h}\right\}$, where $h$ is kept fixed and $p, q \rightarrow \infty$. Also, we consider an $h-p$ version over a quasi-uniform family of meshes $\left\{M^{h}\right\}$, where both $h$ and $p, q$ are changed for accuracy. Then we have the following estimate for $F_{0}$ (Theorem 4.2 of [5]).

Lemma 5.1. Let $\left\{V^{N}\right\}=\left\{V_{p, q}^{i, h}\right\}$ be a sequence of $p$ version spaces on a fixed mesh $M^{h}$, with $p, q \rightarrow \infty$. Then (3.3) and (3.4) (for any fixed $d_{0}>0$ ) are satisfied with

$$
F_{0}(N)=C N^{-(k-1) / 2}
$$

where $C$ is independent of $N$ but depends on $h, k$. Moreover, as $p, q \rightarrow \infty$, (5.1) also holds if the $h-p$ version over a quasi-uniform family of meshes $\left\{M^{h}\right\}$ is used. In this case, we have the following more refined estimate:

$$
F_{0}(N)=C h^{\min (r, k-1)} r^{-(k-1)},
$$

where $r=\min (p, q)$.

Note that in the above, $\left\{M^{h}\right\}$ does not have to be a family of uniform meshes, but can be a quasi-uniform family of meshes.

We now show that there is no locking when the $p$ version is used for our model problem. This result is closely related to a similar result for nearly incompressible materials in $[5,16]$.

Theorem 5.1. Let the extension procedure $\mathscr{F}$ consist of the $p$ version using a mesh consisting of triangles or parallelograms (which can be arbitrary). Then with solution sets $H_{k, d}, k \geq 1, \mathscr{F}$ is free of locking and is robust with uniform order $N^{-(k-1) / 2}$ as $p, q \rightarrow \infty$. 
Proof. Using the results of $[11,15]$, we can show that for the $p$ version with $C_{\text {per }}^{(1)}$ continuous triangular or parallelogram straight-sided elements, for $\omega \in$ $H_{\text {per }}^{k+2}(\Omega), k \geq 1,(h$ fixed $)$, there holds

$$
\inf _{z \in \mathscr{P}_{r, 1,1}^{1, h}}\|\omega-z\|_{2} \leq C r^{-k}\|\omega\|_{k+2} .
$$

Now for any $i=1,2,3$, with $V^{N}=V_{p, q}^{i, h}=\left[\mathscr{P}_{p, 0}^{i, h}\right]^{2} \times \mathscr{P}_{q, 0}^{i, h}$, we have $\mathscr{P}_{r, 1}^{1, h} \subset$ $W^{N}$ for $r=\min (p, q)$. Since $r=O\left(N^{1 / 2}\right)$, we have, with $g(N)$ defined by (3.8),

$$
g(N)=C N^{-k / 2},
$$

so that the method is robust with order $\max \left(g(N), F_{0}(N)\right)=C N^{-(k-1) / 2}$ by Lemma 5.1. The theorem follows, by using Theorem 3.3.

Let us remark that in the above proof, it is observed that $g(N)$ is of a smaller order than $F_{0}(N)$. This implies that for the limiting case (where we have the biharmonic problem), the $p$ version actually shows an increase in the rate of convergence (by one order of $p$ ), rather than a decrease owing to locking. The reason is that the solution to the biharmonic problem is of higher regularity than that of the plate problem, and the asymptotic rate of convergence of the $p$ version only depends upon the regularity of the solution.

For the $h-p$ version, we may show the following theorem for triangular meshes.

Theorem 5.2. Let the extension procedure $\mathscr{F}$ consist of the $h-p$ version, using quasi-uniform meshes consisting of triangles. Let $V^{N}=V_{p, q}^{1, h}$ with $p \geq 4$, $q \geq p+1$. Then with solution sets $H_{k, d}, k \geq 1, \mathscr{F}$ is free of locking and is robust with uniform order $N^{-(k-1) / 2}$ (or, more precisely, $\left.h^{k-1} p^{-(k-1)}\right)$.

Proof. The essential idea is to use the following estimate for the $h-p$ version with $C_{\mathrm{per}}^{(1)}$ continuous triangular elements for $\omega \in H_{\mathrm{per}}^{k+2}(\Omega), k \geq 1$ :

$$
\inf _{z \in \mathscr{P}_{r, 1}^{1, h}}\|\omega-z\|_{2} \leq C h^{k} r^{-k}\|\omega\|_{k+2},
$$

provided $r \geq 5, r \geq k+1$. An analog of (5.3) has been established in [6] for the case of $C^{(0)}$ elements-a similar technique, combining (5.1) with a scaling argument, works for the $C^{(1)}$ case as well. For $V^{N}=V_{p, q}^{1, h}$ with $q \geq p+1$, $p \geq 4$, we have $\mathscr{P}_{p+1,1}^{1, h} \subset W^{N}$. Hence, using (5.3) and Theorem 3.3, we have

$$
g(N)=C h^{k} p^{-k}=C N^{-k / 2}
$$

in (3.8). The theorem follows easily.

We see from the above that when the $h-p$ version is used, the separate robustness rates of the $h$ and $p$ versions are combined. Theorem 5.2 therefore combines the results of Theorems 4.1 and 5.1, and says that if the $p$ version is combined with a locking-free $h$ version, then the resulting $h-p$ extension procedure is also free from locking.

Remark 5.1. As mentioned in the introduction, an alternating analysis of shear locking is based on the Helmholtz decomposition of the shear strain,

$$
d^{-2}\left(\nabla \omega_{d}-\vec{\phi}_{d}\right)=\nabla r_{d}+\nabla \times p_{d}
$$


(see $[1,9,10])$. This reduces $(2.1)-(2.2)$ to a Stokes-like system for $\left(\vec{\phi}_{d}, p_{d}\right)$ and uncoupled Poisson equations for $r_{d}$ and $\omega_{d}$. The locking effects are related to the Stokes-like system. It is possible to derive an analog of condition $(\alpha)$ for this system and then develop the locking theory in the previous sections via this approach.

As is well known, the Stokes problem is the limit (as the Poisson ratio $\nu \rightarrow 0.5$ ) of the equations of linear elasticity for an almost incompressible material. The question of locking for nearly incompressible materials has been well studied in the literature (see, e.g., $[5,13,14,16])$. This theory could essentially be applied to the Stokes-like system for $\left(\vec{\phi}_{d}, p_{d}\right)$, which behaves similarly, since it tends to the same limit. Hence, spaces $Y^{N}$ that are locking-free for the displacements in nearly incompressible elasticity (as analyzed in $[5,13,14,16]$ ) will behave similarly for the rotations $\vec{\phi}_{d}$ in the RM plate. (Of course, they must now be combined with appropriate subspaces $Z^{N}$ for the transverse displacements $\omega_{d}$. .) In this way, many of our results for the RM plate established here may be predicted from the results for nearly incompressible materials in the above references.

\section{The $(1,1,2)$ Plate MOdel AND hieraRCHiCAL MOdELING}

So far, we have only considered the RM model and its zero thickness limit, the Kirchhoff model, for modeling the actual 3-d plate.

In this section, our goal is to analyze the locking characteristics of higherorder models, particularly the $(1,1,2)$ model. The derivation of the $(1,1,2)$ model and the related hierarchy is based on the "expansion" of the exact 3-d solution in terms of polynomials in the transverse direction. More precisely, let $\vec{u}=\left(u_{1}, u_{2}, u_{3}\right)$ denote the displacement of the plate occupying the region $R=\bar{\Omega} \times\left[-\frac{d}{2}, \frac{d}{2}\right]$ described in $\S 2$, along the coordinate axes. For any $n=$ $\left(n_{1}, n_{2}, n_{3}\right), n_{i} \geq 0$ integer, we define

$$
\begin{array}{r}
{ }^{n} H_{\mathrm{per}}^{1}=\left\{\vec{u} \in\left[H_{\mathrm{per}}^{1}(R)\right]^{3} \mid u_{i}=\sum_{j=0}^{n_{i}} \phi_{i j}\left(x_{1}, x_{2}\right) L_{j}\left(\frac{x_{3}}{d / 2}\right), i=1,2,\right. \\
\left.u_{3}=\sum_{j=0}^{n_{3}} \omega_{j}\left(x_{1}, x_{2}\right) L_{j}\left(\frac{x_{3}}{d / 2}\right)\right\},
\end{array}
$$

where $L_{j}(x)$ are Legendre polynomials. Suppose, for any displacement $\vec{v} \in$ $\left[H_{\text {per }}^{1}(R)\right]^{3}, \mathscr{G}(\vec{v})$ represents the corresponding plate energy, so that the exact 3-d solution $\vec{u}_{3 d}$ is the minimizer of $\mathscr{G}(\vec{v})$ over all such $\vec{v} \in\left[H_{\mathrm{per}}^{1}(R)\right]^{3}$. Then $\vec{u}^{n} \in{ }^{n} H_{\text {per }}^{1}$, the solution of the $\left(n_{1}, n_{2}, n_{3}\right)$ plate model, is defined as the unique minimizer of $\mathscr{G}(\vec{v})$ over all $\vec{v} \in{ }^{n} H_{\mathrm{per}}^{1} \subset\left[H_{\mathrm{per}}^{1}(R)\right]^{3}$. Allowing $n$ to vary then gives a hierarchy of plate models. For fixed $d>0, \vec{u}^{n} \rightarrow \vec{u}_{3 d}$ (the actual 3-d plate model displacement) as $n \rightarrow \infty$. Also, as $d \rightarrow 0, \vec{u}^{n} \rightarrow \vec{u}_{3 d}$ at a higher rate in $d$ when $n$ is increased and $\vec{u}_{3 d}$ is regular (see [12] for more details).

Since we are considering the case of pure bending (which exhibits shear locking), we may take $\phi_{1 j}=\phi_{2 j}=0$ for $j$ even, $\omega_{j}=0$ for $j$ odd. The simplest model in this hierarchy is then obtained by taking $n=(1,1,0)$, which yields 
the three unknowns, $\phi_{10}, \phi_{20}, \omega_{0}$ over the region $\Omega$. This model is equivalent to the RM plate model (2.1)-(2.2), with $\phi_{d i} \equiv-\frac{2}{d} \phi_{i 0}, i=1,2$ and $\omega_{d} \equiv \omega_{0}$ (provided the elastic constants are suitably modified (see [2])).

The model we discuss here is the next higher model in the hierarchy, obtained by choosing $n=(1,1,2)$. This gives four unknown fields over $\Omega$, namely $\phi_{10}, \phi_{20}, \omega_{0}$ and $\omega_{2}$. Denoting $u_{d}=\left(\vec{\phi}_{d}, \omega_{d}, y_{d}\right)$, where $\phi_{d 1}, \phi_{d 2}, \omega_{d}$ are as defined above and $y_{d} \equiv \omega_{2}$, we may write the Euler equation for the $(1,1,2)$ model (analogous to $(2.1)-(2.2))$ as follows:

$$
\begin{gathered}
\widetilde{C}_{d} u_{d}=-d^{2} \frac{D}{2}\left\{(1-\nu) \Delta \vec{\phi}_{d}+\frac{(1-\nu)}{(1-2 \nu)} \nabla \nabla \cdot \vec{\phi}_{d}\right\} \\
-\mu\left(\nabla \omega_{d}-\vec{\phi}_{d}\right)+\lambda \nabla y_{d}=0, \\
-\mu d^{-2} \nabla \cdot\left(\nabla \omega_{d}-\vec{\phi}_{d}\right)=g, \\
-\frac{\mu d^{-2}}{5} \Delta y_{d}-\lambda d^{-2} \nabla \cdot \vec{\phi}_{d}+12 d^{-4}(2 \mu+\lambda) y_{d}=g .
\end{gathered}
$$

Here, the elastic constants are the same as defined in $\S 2$, and periodic boundary conditions are assumed for $u_{d}$.

We may combine (6.3)-(6.4) to obtain the following equation instead of (6.4):

$$
\widetilde{B}_{d} u_{d}=y_{d}+\frac{d^{2}}{12(2 \mu+\lambda)}\left(\mu \nabla \cdot\left(\nabla \omega_{d}-\vec{\phi}_{d}\right)-\frac{\mu}{5} \Delta y_{d}-\lambda \nabla \cdot \vec{\phi}_{d}\right)=0 .
$$

For $d>0$, it has been shown in [2] that the $(1,1,2)$ model exhibits higher accuracy than the RM model for several quantities of engineering interest. As $d \rightarrow 0$, the two models both tend to the Kirchhoff plate. Using (6.5), we see that in the limit,

$$
\widetilde{B}_{0} u_{0}=y_{0}=0,
$$

so that using (6.6) in the limit $\widetilde{C}_{0} u_{0}=0$ of (6.2) shows once again that (2.4) holds. Moreover, it may be verified that $\omega_{0}$ will again satisfy (2.5), with periodic boundary conditions.

The weak form of (6.2)-(6.4) is as follows. For $d \in(0,1]$, find $u_{d}=$ $\left(\vec{\phi}_{d}, \omega_{d}, y_{d}\right) \in V=\left[H_{\mathrm{per}}^{1}(\Omega)\right]^{4}$ such that for all $v=(\vec{\theta}, \xi, z) \in V$,

$$
\begin{aligned}
a_{d}\left(u_{d}, v\right)= & \tilde{b}\left(u_{d}, v\right)+\frac{\mu}{d^{2}}\left\langle C_{0} u_{d} C_{0} v\right\rangle+\frac{\lambda}{d^{2}}\left(\left\langle\vec{\theta}, \nabla y_{d}\right\rangle+\left\langle\vec{\phi}_{d}, \nabla z\right\rangle\right) \\
& +\frac{\mu}{5 d^{2}}\left(\nabla y_{d}, \nabla z\right)+\frac{12}{d^{4}}(2 \mu+\lambda)\left\langle y_{d}, z\right\rangle=F(v) .
\end{aligned}
$$

Here, $C_{0}$ is as defined in (2.4),

$$
\tilde{b}\left(u_{d}, v\right)=\frac{D}{2}(1-\nu) \iint_{\Omega}\left\{\nabla \vec{\phi}_{d} \cdot \nabla \vec{\theta}+\frac{1}{(1-2 \nu)}\left(\nabla \cdot \vec{\phi}_{d}\right)(\nabla \cdot \vec{\theta})\right\} d x_{1} d x_{2}
$$

and

$$
F(v)=\langle g, \xi\rangle+\langle g, z\rangle=\iint_{\Omega} g(\xi+z) d x_{1} d x_{2} .
$$

Suppose now that we are given a sequence $\left\{V^{N}\right\}$ of finite-dimensional subspaces of $V=\left[H_{\text {per }}^{1}(\Omega)\right]^{4}$. Then the finite element solutions $u_{d}^{N} \in V^{N}$ may 
be defined again by (3.1). As $d \rightarrow 0$, assuming that $a_{d}\left(u_{d}^{N}, u_{d}^{N}\right)$ is bounded independently of $d$ will constrain various terms in (6.7) involving $u_{d}^{N}$. Our goal is to show that these constraints cause exactly the same type of locking as in the RM case, and that the locking-free subspaces constructed for (2.8) yield corresponding locking-free subspaces for (6.7) when augmented by a suitable subspace for the additional variable $y_{d}$.

We first show that condition $(\alpha)$ holds for the $(1,1,2)$ model. To do this, we modify the definitions of various spaces introduced in $\S 2$, in order to incorporate the extra unknown $y_{d}$. We let $H_{\mathrm{per}}^{k, l, m}(\Omega)=H_{\mathrm{per}}^{k, l}(\Omega) \times H_{\mathrm{per}}^{k}(\Omega)$. Let $\|\cdot\|_{E, d}$ be defined by (2.9) and $\|\cdot\|_{k, l, m}$ by the analog of (2.10). Then, (2.11) will once again hold, except that $\beta=2$ now, i.e., an extra power of $d^{-1}$ appears.

The spaces $H_{k, d}$ are now given by

$$
H_{k, d}=\left\{u \in H_{\mathrm{per}}^{k+1, k, k}(\Omega), \widetilde{C}_{d} u=0, \widetilde{B}_{d} u=0\right\},
$$

which once again can be characterized in terms of $\vec{\phi}$ alone, as in (2.15). The space $H_{k, d}^{B}$ is now defined as follows:

$$
H_{k, d}^{B}=\left\{u \in H_{k, d},\|u\|_{k, d} \leq B\right\},
$$

where

$$
\|u\|_{k, d}^{2}=\|(\vec{\phi}, \omega, y)\|_{k, d}^{2}=\|\vec{\phi}\|_{k+1}^{2}+\|\omega\|_{k}^{2}+d^{-2}\|y\|_{k}^{2} .
$$

Then we see that for $u=(\vec{\phi}, \omega, y) \in H_{k, d}^{B}$, by (6.10)-(6.11), we have

$$
\left\|B_{0} u\right\|_{k}=\|y\|_{k} \leq d^{2} B \text {. }
$$

Using (6.12) and $\widetilde{C}_{d} u=0$ then gives (2.16) as before.

The limiting set of $H_{k, d}$ is now given by

$$
H_{k, 0}=\left\{u=(\vec{\phi}, \omega, 0),(\phi, \omega) \in H_{\mathrm{per}}^{k+1, k+2}(\Omega), C_{0} u=0\right\}
$$

with $H_{k, 0}^{B}$ being the analog of $(2.17 \mathrm{~b})$. Then the following theorem holds.

Theorem 6.1. Let $g$ be as in Theorem 2.1. Then there exists a unique sequence of periodic solutions $\left\{u_{d}\right\}=\left\{\vec{\phi}_{d}, \omega_{d}, y_{d}\right\}$ ( $\omega_{d}$ unique up to a constant) to (6.2)(6.4) for $d \in(0,1]$ and (2.3)-(2.5), (6.6) for $d=0$, such that $u_{d} \in H_{s, d}^{B}$, where $B=C\|g\|_{s-2}$, with $C$ a constant independent of $g$ and $d$.

Proof. Analogously to (2.19), we may now show that the solution of (6.2)-(6.4) is given by (see [12])

$$
\begin{aligned}
u_{d} & =\left(\begin{array}{l}
\phi_{d 1} \\
\phi_{d 2} \\
\omega_{d} \\
y_{d}
\end{array}\right) \\
& =\sum_{k \in \mathbb{Z}_{0}^{2}} A_{k} e^{i k \cdot x}\left(\begin{array}{c}
\frac{i k_{1} 3(\nu-1)}{4}\left(30+3 d^{2}|k|^{2}\right) \\
\frac{i k_{2} 3(\nu-1)}{4}\left(30+3 d^{2}|k|^{2}\right) \\
\frac{1}{4}\left(\left(90+\frac{d^{4}}{8}|k|^{4}\right)(\nu-1)+\frac{d^{2}|k|^{2}}{4}(36 \nu-63)\right) \\
\frac{5 d^{2}|k|^{2}}{8}\left(3 \nu+\frac{d^{2}|k|^{2}}{4}(\nu-1)\right)
\end{array}\right),
\end{aligned}
$$


where $A_{k}=g^{k} / \mu|k|^{4}\left((\nu-1) \frac{d^{2}}{4}|k|^{2}-30\right)$. (Equation (6.13) was derived from (6.2)-(6.4) using a computer algebra system.) The proof then follows similarly to the proof of Theorem 2.1.

The rate at which the solution of the $(1,1,2)$ model tends to the limiting solution of the Kirchhoff plate in the $H_{\text {per }}^{s+1, s, s}(\Omega)$ norm (condition $(\alpha)$ ), is given in the following analog of Theorem 2.2.

Theorem 6.2. For any $u_{d}=\left(\vec{\phi}_{d}, \omega_{d}, y_{d}\right) \in H_{s, d}^{B}$, there exists $u_{0}=\left(\vec{\phi}_{0}, \omega_{0}, 0\right)$ $\in H_{s, 0}^{c B}$ such that

$$
\left\|u_{d}-u_{0}\right\|_{s+1, s, s} \leq K d^{2} B
$$

where $c$ and $K$ are constants independent of $u_{d}, d, B$.

Proof. Given $u_{d} \in H_{s, d}^{B}$, define $g$ satisfying (2.21) as in Theorem 2.2; also, let $\omega_{0}$ be given by (2.20) and $\vec{\phi}_{0}=\omega_{0}$. Then (6.14) may be verified using (2.19), (2.20) and (6.12).

Finally, let us note that the analog of Theorem 2.3 for the $(1,1,2)$ model is easily established as well.

We may now formally define locking and robustness for the $\|\cdot\|_{1,1,1}$ or $\|\cdot\|_{E, d}$ norm, as before. Our space $V^{N}=Y^{N} \times Z^{N} \times M^{N}$ will be assumed to be $F_{0}$-admissible in the sense analogous to (3.3). (This means that for any spaces $Y^{N}, Z^{N}$ for the RM plate, we can construct $V^{N}$ by adding a space $M^{N}$ that approximates the unknown $y_{d}$ in the $H_{\mathrm{per}}^{1}(\Omega)$ norm at the rate $F_{0}(N)$.) Then the following analog of Theorem 3.3 will hold.

Theorem 6.3. Let the spaces $V^{N}=Y^{N} \times Z^{N} \times M^{N}$ be $F_{0}$-admissible for the $(1,1,2)$ model. Let $W^{N}$ be as in Theorem 3.3. Then the extension procedure $\mathscr{F}=\left\{V^{N}\right\}$ is robust with uniform order $\max \left\{F_{0}(N), g(N)\right\}$, where $g(N)$ is given by (3.8). Moreover, $\mathscr{F}$ is free from locking if and only if (3.9) holds. It shows locking of order $f(N)$ if and only if (3.10) holds.

The proof of Theorem 6.3 is analogous to that of Theorem 3.3. The only difference is that in (3.12), $v_{1}$ must now satisfy $\widetilde{B}_{0} v_{1}=0$ as well. Also here, $\beta=2$, so that both powers of $d$ in condition $(\alpha)$ (i.e., (6.14)) are used.

We therefore see that taking (for example) $M^{N} \equiv Y^{N}$ leads to the same locking and robustness results for the $(1,1,2)$ model as described in $\S \S 4$ and 5 for the RM model.

A similar analysis (using more notation) may be done for higher-order models as well. Essentially, the higher-order terms in the expansion of the 3-d solution all are constrained to zero in the limiting case (similar to $y_{0}$ ) and the only constraint that causes locking is the Kirchhoff constraint. Hence, the locking and robustness properties of various subspaces can be once again characterized by the results in $\S \S 4$ and 5 .

Remark 6.1. From (6.2), it is observed that the $(1,1,2)$ model will also exhibit "Poisson ratio locking" when $\nu$ is close to 0.5 . This locking does not occur for the RM plate, owing to the elastic constants being modified, but will occur for all higher-order models. By Remark 5.1, we see, however, that an element that is free of shear-locking (in the energy) as $d \rightarrow 0$ will also be free of Poisson locking as $\nu \rightarrow 0.5$, so that no additional precautions need to be taken to prevent Poisson locking. 


\section{BIBLIOGRAPHY}

1. D. N. Arnold and R. S. Falk, A uniformly accurate finite element method for the Reissner-Mindlin plate, SIAM J. Numer. Anal. 26 (1989), 1276-1290.

2. I. Babuška and $\mathrm{L}$. Li, The problem of plate modeling. Theoretical and computational results, Comput. Methods Appl. Mech. Engrg. 100 (1992), 249-273.

3. I. Babuška and J. Pitkäranta, The plate paradox for hard and soft simple support, SIAM J. Math. Anal. 21 (1990), 551-576.

4. I. Babuška and M. Suri, On locking and robustness in the finite element method, SIAM J. Numer. Anal. 29 (1992), 1261-1293.

5. $\ldots$, Locking effects in the finite element approximation of elasticity problems, Numer. Math. 62 (1992), 439-463.

6. $\ldots$ The $h-p$ version of the finite element method with quasi-uniform meshes, RAIRO Modél. Math. Anal. Numér. 21 (1987), 199-238.

7. J. Bergh and J. Löfström, Interpolation spaces, Springer-Verlag, Berlin, 1976.

8. C. de Boor and R. A. DeVore, Approximation by smooth multivariate splines, Trans. Amer. Math. Soc. 276 (1983), 775-788.

9. F. Brezzi and M. Fortin, Mixed and hybrid finite element methods, Springer-Verlag, New York, 1991.

10. F. Brezzi, M. Fortin, and R. Stenberg, Error analysis of mixed-interpolated elements for Reissner-Mindlin plates, Math. Models Methods Appl. Sci. 1 (1991), 125-151.

11. S. Jensen, $A n H_{0}^{m}$ interpolation result, SIAM J. Math. Anal. 22 (1991), 785-791.

12. C. Schwab, The Fourier approach to the asymptotic analysis of plate models, Technical Report 93-4, University of Maryland Baltimore County, Baltimore, MD, 1993.

13. L. R. Scott and M. Vogelius, Norm estimates for a maximal right inverse of the divergence operator in spaces of piecewise polynomials, RAIRO Modél. Math. Anal. Numér. 19 (1985), 111-143.

14. __ Conforming finite element methods for incompressible and nearly incompressible continua, Large Scale Computations in Fluid Mechanics, Lectures in Appl. Math., vol. 22, Part 2, Amer. Math. Soc., Providence, RI, 1985, pp. 221-244.

15. M. Suri, The $p$ version of the finite element method for elliptic equations of order $2 l$, RAIRO Modél. Math. Anal. Numér. 24 (1990), 265-304.

16. M. Vogelius, An analysis of the p-version of the finite element method for nearly incompressible materials, Uniformly valid, optimal error estimates, Numer. Math. 41 (1983), 19-37.

(Suri and Schwab) Department of Mathematics and Statistics, University of Maryland

Baltimore COUNTY, BALTIMORE, MARYland 21218

E-mail address, M. Suri: suri@umbc2 .umbc.edu

E-mail address, C. Schwab: schwab@math.umbc.edu

(Babuška) Institute of Physical Science and Technology, University of Maryland, College Park, Maryland 20740 